\title{
ELECTRON-PHONON COUPLING IN RARE EARTH COMPOUNDS
}

\author{
A. Meijerink, G. Blasse, J. Sytsma, C. de Mello Donega \\ AND A. Ellens \\ Debye Institute, University Utrecht \\ P.O. Box 80 000, 3508 TA Utrecht, The Netherlands
}

The variation in electron-phonon coupling strength for rare earth ions has been studied by measuring vibronic transition probabilities and temperature dependent line broadening for a large number of rare earth ions in different liost lattices. The results show a large variation in the electron-phonon coupling strength (almost three orders of magnitude) and a remarkable trend: the coupling is strong for ions in the beginning and at the end of the lanthanide series and weak in the middle. In covalent host lattices the coupling is much stronger than in ionic lost lattices. An explanation of the results is given and the consequences of the variation of the electron-phonon coupling strength on phenomena like multiphonon relaxation, energy transfer and cooperative luminescence are discussed.

PACS numbers: $33.70 . \mathrm{Jg}, 33.50 . \mathrm{Dq}, 33.50 . \mathrm{Hv}$

\section{Introduction}

The shielding of the $4 f$ electrons by the outer $5 s^{2}$ and $5 p^{6}$ electrons give the lanthanides their unique luminescence spectra dominated by sharp zero-phonon lines. These characteristic spectra resemble spectra found in atomic (gas) spectroscopy. It is well known that the coupling of the $4 f$ electrons with their surroundings is weak due to the shielding. Still, many important phenomena like multiphonon relaxation, (phonon assisted) energy transfer, cooperative luminescence and cross relaxation are only possible due to interaction of the $4 f$ electrons and their surroundings. Until recently, systematic studies on the variation of the electron-phonon coupling strength for lanthanides have been scarce [1-3]. In a few studies the variation of the electron-phonon coupling strength for rare earth ions have been studied. The coupling has been predicted to be approximately the same for all rare earths, to decrease through series, or to be strong in the beginning and at the end of the series and weak in the middle. Also for the host lattice dependence conflicting ideas have been proposed: both weaker and stronger coupling for rare earth ions in covalent host lattices have been reported $[4,5,7]$. 
In this paper an overview will be given of eight years of systematic research in our group on the electron-phonon coupling strength for rare earth ions [6-9]. Two methods have been used to probe the variation in the electron-phonon coupling strength: vibronic transition probabilities and temperature dependent line broadening. Vibronic transition probabilities have been determined for light $\left(\mathrm{Pr}^{3+}\right)$, middle $\left(\mathrm{Gd}^{3+}\right)$ and heavy $\left(\mathrm{Tm}^{3+}\right)$ lanthanides. The results show a large variation in the electron-phonon coupling strength, with the strongest coupling for the heavy and light lanthanides. A systematic study of the temperature dependent line broadening for transitions within the $4 f^{n}$ configuration of a large number of lanthanides (viz. $\mathrm{Ce}^{3+}, \mathrm{Pr}^{3+}, \mathrm{Nd}^{3+}, \mathrm{Eu}^{3+}, \mathrm{Gd}^{3+}, \mathrm{Tb}^{3+}, \mathrm{Er}^{3+}, \mathrm{Tm}^{3+}$ and $\mathrm{Yb}^{3+}$ ) in $\mathrm{LiYF}_{4}$ confirms this trend: the electron-phonon coupling is strong in the beginning and at the end of the lanthanide series and weak in the middle. A qualitative explanation for this trend will be given in terms of lanthanide contraction, shielding factor for the $4 f$ electrons and the position of the opposite parity state.

The influence of the host lattice on the electron-phonon coupling strength has been studied by comparing vibronic transition probabilities and temperature dependent line broadening for various lanthanide ions in $\mathrm{La}_{2} \mathrm{O}_{3}$ and $\mathrm{LiYF}_{4}$. The results show that the electron-phonon coupling is considerably stronger in a covalent host lattice $\left(\mathrm{La}_{2} \mathrm{O}_{3}\right)$.

In the last section of this paper the consequences of the large variation of the electron-phonon coupling strength for rare earth ions in the phenomena like multiphonon relaxation, cooperative luminescence and energy transfer will be discussed for different rare earth ions in different host lattices.

\section{Vibronic transition probabilities}

Vibronic transitions are due to a combined electronic and vibrational transition. In the luminescence spectra of rare earth ions they can be observed as

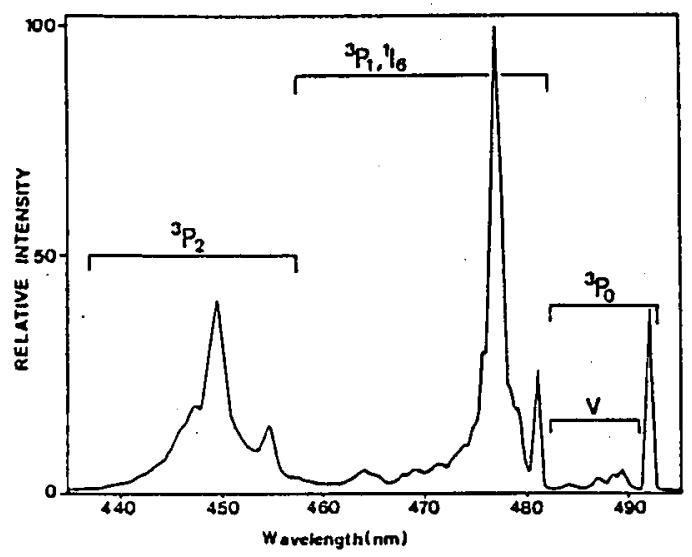

Fig. 1. Excitation spectrum of the ${ }^{3} P_{0} \rightarrow{ }^{3} F_{2}$ emission of $\mathrm{Pr}^{3+}$ in $\mathrm{La}_{2} \mathrm{O}_{3}$ in the ${ }^{3} H_{4} \rightarrow{ }^{3} P_{J}$ region at $4 \mathrm{~K}$. For the ${ }^{3} H_{4} \rightarrow{ }^{3} P_{0}$ transition vibronic lines are indicated by $v$. 
weak side bands next to strong zero-phonon lines. In Fig. 1 an example of vibronic lines is given. The intensity of the vibronic lines depends on the coupling of the $4 f$ electrons with their environment: stronger coupling leads to stronger vibronic lines. A good measure of the intensity of vibronic lines is the integrated transition probability for vibronic side bands for an intraconfigurational $4 f^{n}$ transition. Vibronic transition probabilities can be determined by measuring the intensity of the vibronic lines relative to the zero-phonon line and the radiative transition probability for the zero-phonon line which can be obtained from decay time measurements. In Ref. [7] the procedure to determine vibronic transition probabilities is discussed in detail. For rare earth ions the vibronic transition probabilities have been determined for $\mathrm{Pr}^{3+}, \mathrm{Gd}^{3+}$ and recently also $\mathrm{Tm}^{3+}$ [6-8]. For $\mathrm{Gd}^{3+}$ and $\mathrm{Pr}^{3+}$ the results are relatively accurate since vibronic lines can be observed next to one zero-phonon line of a transition to a non-degenerate level $\left({ }^{6} P_{7 / 2} \rightarrow{ }^{8} S_{7 / 2}\right.$ for $\mathrm{Gd}^{3+}$ and ${ }^{3} H_{1} \rightarrow{ }^{3} P_{0}$ for $\mathrm{Pr}^{3+}$ ). For other rare earth ions the analysis of vibronic transition probabilities is complicated by the crystal field splitting of the final level. The crystal field splitting is of the same order of magnitude as the vibrational energies. Overlap between vibronic lines and the strong zero-phonon lines due to transitions to different crystal field components makes it hard to determine vibronic transition probabilities. Still, for $\mathrm{Tm}^{3+}$ it was possible to estimate vibronic transition probabilitics [8].

\section{TABLE I}

Vibronic transition probabilities for $\mathrm{Pr}^{3+}\left(4 f^{2}\right), \mathrm{Gd}^{3+}$ $\left(4 f^{7}\right)$ and $\mathrm{Tm}^{3+}\left(4 f^{12}\right)$ in different host lattices. The values are determined for the ${ }^{3} H_{4} \rightarrow{ }^{3} P_{0}\left(\mathrm{Pr}^{3+}\right)$, the ${ }^{6} P_{7 / 2} \rightarrow{ }^{8} S_{7 / 2}\left(\mathrm{Cid}^{3+}\right)$ and the ${ }^{1} D_{2} \rightarrow{ }^{3} F_{4}\left(\mathrm{Tm}^{3+}\right)$ transitions.

\begin{tabular}{l|c|c|c}
\hline \hline Host lattice & $\begin{array}{c}\mathrm{Pl}^{3+} \\
A_{\text {vib }}\left[\mathrm{s}^{-1}\right]\end{array}$ & $\begin{array}{c}\mathrm{Gd}^{3+} \\
A_{\text {vib }}\left[\mathrm{s}^{-1}\right]\end{array}$ & $\begin{array}{c}\mathrm{Tm}^{3+} \\
A_{\text {vib }}\left[\mathrm{s}^{-1}\right]\end{array}$ \\
\hline $\mathrm{LiYF}_{4}$ & 100 & 6 & 250 \\
$\mathrm{Na}_{5} \mathrm{La}\left(\mathrm{WO}_{4}\right)_{4}$ & 1400 & - & 2000 \\
$\mathrm{YOCl}$ & 4600 & 114 & 2300
\end{tabular}

In Table I some results on vibronic transition probabilities are presented for $\mathrm{Pr}^{3+}, \mathrm{Gd}^{3+}$ and $\mathrm{Tm}^{3+}$ in different host lattices. Comparison of the vibronic transition probabilities shows that the values for $\mathrm{Gd}^{3+}$ are small and that those for $\mathrm{Tm}^{3+}$ and $\mathrm{Pr}^{3+}$ are larger. For the same rare earth ion in different host lattices the vibronic transition probabilities increase when the host lattice is more covalent: the smallest values are found for rare earth ions in $\mathrm{LiYF}_{4}$ and highest vibronic transition probabilities are found for rare earth ions in the covalent host lattice YOCl. More results on vibronic transition probabilities for $\mathrm{Pr}^{3+}, \mathrm{Gd}^{3+}$ and $\mathrm{Tm}^{3+}$ can be found in Refs. [6-8].

The variation of the vilbronic transition probabilities is very large: for $\mathrm{Gd}^{3+}$ in $\mathrm{LiYF}_{4}$ a value $6 \mathrm{~s}^{-1}$ is found. For $\mathrm{Pr}^{3+}$ and $\mathrm{Tm}^{3+}$ in a covalent host lattice 
the values are almost three orders of magnitude higher. These results show that the weak electron-phonon coupling can vary considerably depending on the type of rare earth ion and the host lattice. An explanation for the variation of the electron-phonon coupling strength will be given at the end of the next section on line broadening.

\section{Line broadening}

A second method to probe the electron-phonon coupling strength for rare earth ions is temperature dependent line broadening. At elevated temperatures the line width of the zero-phonon lines increases due to phonon induced relaxation processes. Different relaxation processes can contribute to the shortening of the lifetime of both the initial and the final level of a transition, for example one-phonon emission and absorption, two-phonon Raman processes, multiphonon relaxation and Orbach processes [10]. The line width of a transition between the initial and final level increases (according to the Ileisenberg uncertainty principle) if the lifetimes of the initial and final levels become shorter. Temperature dependent line width measurements can provide information on the electron-phonon coupling strength since stronger electron-phonon coupling will result in more pronounced line broadening. As an example of line broadening the emission spectra of the ${ }^{2} F_{5 / 2} \rightarrow{ }^{2} F_{7 / 2}$ transition on $\mathrm{Yb}^{3+}$ in LiYF 4 are shown in Fig. 2. Up to $20 \mathrm{~K}$ the zero-phonon line has a Gaussian line shape due to inhomogeneous broadening. At higher temperatures the line width starts to increase and the line shape changes from Gaussian to Lorentzian, as is expected for a homogeneously (lifetime) broadened line. The drawn lines at $20 \mathrm{~K}$ and $80 \mathrm{~K}$ in Fig. 2 are fits to a Gaussian and Lorentzian line shape, respectively.

Theoretical and experimental work in the past decades has shown that for most cases of the line broadening for rare earth ions the Raman two-phonon process

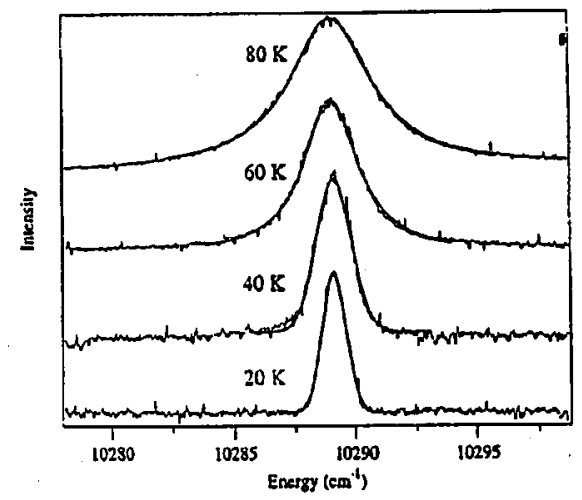

Fig. 2. Emission spectra of the ${ }^{2} F_{5 / 2} \rightarrow{ }^{2} F_{7 / 2}$ transition of $\mathrm{Yb}^{3+}$ in $\mathrm{LiYF}_{4}$ at 20,40, 60 and $80 \mathrm{~K}$ for excitation in the ${ }^{2} F_{5 / 2}$ level. 


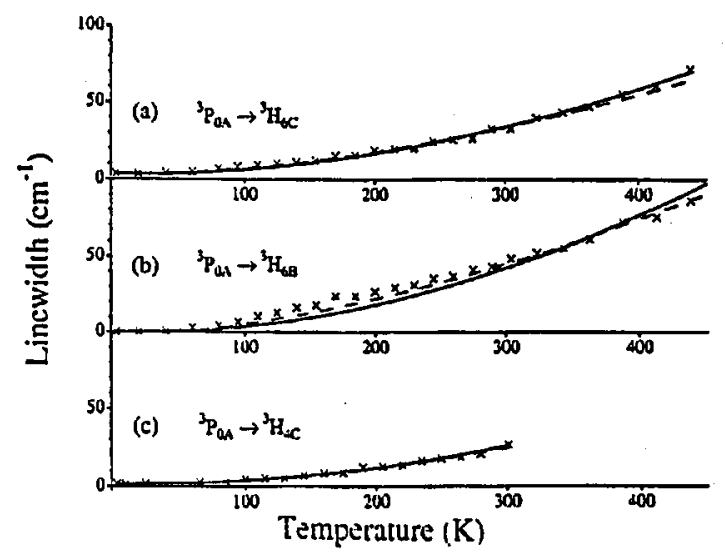

Fig. 3. Fits and simulations to the temperature dependence of the line width of different transitions on $\mathrm{Pr}^{3+}$ in $\mathrm{LiYF}_{4}$. The transitions are indicated in the figure. The drawn lines are fits to Eq. (2) (Rainan process only) and the broken lines are simulations which also include one-phonon (direci) relaxation processes.

is dominant, although also one-phonon relaxation processes (also called direct processes) can have a significant contribution $[11,12]$. The temperature dependence of the line width for the Raman process is given by

$$
\Delta E^{\mathbf{R}}(T)=\bar{\alpha}\left(\frac{T}{T_{\mathrm{D}}}\right)^{7} \int_{0}^{\frac{T_{\mathrm{D}}}{T}} \frac{x^{6} \mathrm{e}^{x}}{\left(\mathrm{e}^{x}-1\right)^{2}} \mathrm{~d} x
$$

In this equation $\bar{\alpha}$ is a parameter for the electron-phonon coupling strength, $T_{D}$ is the effective Debye temperature and $x=\hbar \omega / k T$. In Fig. 3 the temperature dependence of the line width for ${ }^{3} P_{0} \rightarrow{ }^{3} H_{4}$ and ${ }^{3} P_{0} \rightarrow{ }^{3} H_{6}$ transitions on $\mathrm{Pr}^{3+}$ in $\mathrm{LiYF}_{4}$ are shown as examples. The drawn lines are fits to the equation

$$
\Delta E(T)=\Delta E^{\text {inh }}+\Delta E^{\mathrm{R}}(T) .
$$

Here $\Delta E^{\text {inh }}$ is the inhomogeneous line width. The broken lines are simulations in which also one-phonon relaxation processes to a large number of crystal field components within the phonon energy range are included. The contribution of the one-phonon processes is small. A good estimate for the electron-phonon coupling strength can be obtained by fitting the line broadening data to Eq. (2) in which only the dominant two-phonon Raman process is included. The electron-phonon coupling parameters $\bar{\alpha}$ have been determined for a large number of transitions on nine rare earth ions in $\mathrm{LiYF}_{4}$, three in the beginning of the series $\mathrm{Ce}^{3+}, \mathrm{Pr}^{3+}$ and $\left.\mathrm{Nd}^{3+}\right)$, three in the middle $\left(\mathrm{Eu}^{3+}, \mathrm{Gd}^{3+}\right.$ and $\left.\mathrm{Tb}^{3+}\right)$ and three at the end $\left(\mathrm{Er}^{3+}\right.$, $\mathrm{Tm}^{3+}$ and $\mathrm{Yb}^{3+}$ ). The results are compiled in Fig. 4. In this figure a clear trend in the variation of the electron-phonon coupling strength for rare earth ions can be observed. The coupling is strong in the beginning and at the end of the series and weak in the middle. This observation is in agreement with the results on vibronic transition probabilities for $\mathrm{Pr}^{3+}, \mathrm{Gd}^{3+}$ and $\mathrm{Tm}^{3+}$ reported in the previous section. 
La Ce Pr Nd Pm Sm Eu Gd Tb Dy Ho Er Tm Yb Lu

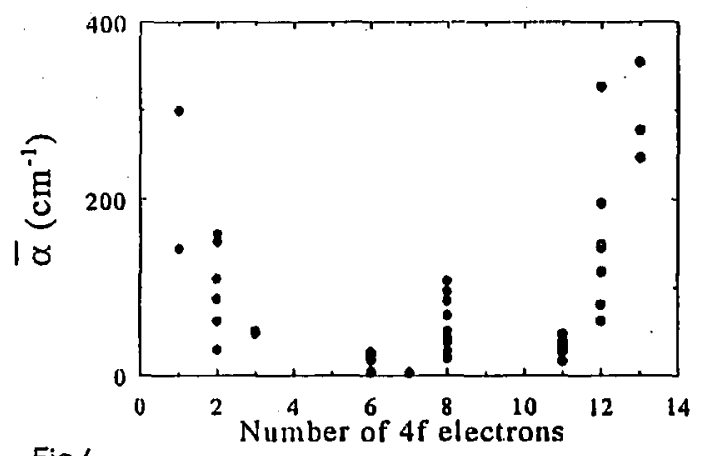

Fig.4

Ce Pr Nd Pm Sm Eu Gd To Dy Ho Er Tm Yb

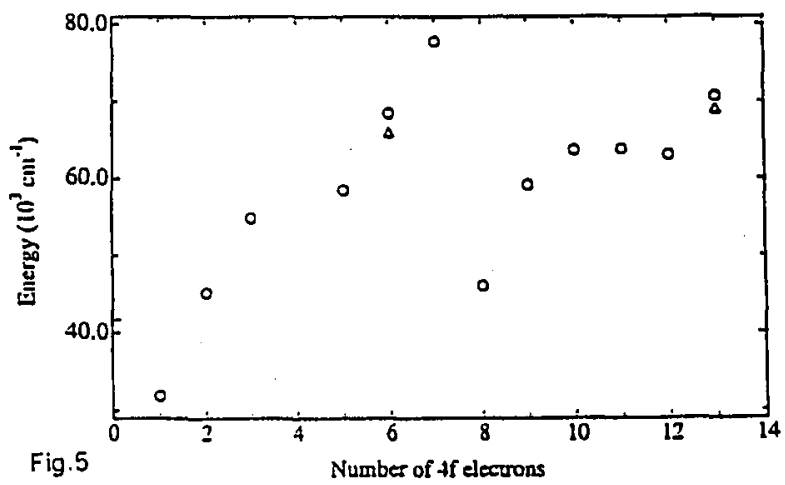

Fig. 4. The electron-phonon coupling parameter $\bar{\alpha}$ for different rare earth ions. Each point gives the $\bar{\alpha}$ value derived from the temperature dependence of the line width of one transition.

Fig. 5. Position of the lowest opposite parity states for rare earth ions in fluorides. The circles give the positions of the lowest $4 f^{n-1} 5 d$ states and the triangles for the charge transfer state. The data were obtained from Ref. [13].

To explain this variation in electron-phonon coupling strength two effects are consiclered:

1) Due to the lanthanide contraction the $4 f$ orbitals are contracted and the overlap between the $4 f$ orbitals and their environment will decrease for heavier lanthanides.

2) The shielding of the $4 f$ electrons by the $5 s^{2}$ and $5 p^{6}$ electrons varies through the lanthanide series.

Blok and Shirley have reported a decrease in the shielding parameter $\sigma_{2}$ from $\mathrm{Ce}^{3+}\left(\sigma_{2}=1.1\right)$ to $\mathrm{Yb}^{3+}\left(\sigma_{2}=0.6\right)$. The smaller screening factors for the $4 f$ electrons of the heavier lanthanides gives rise to an increase in electron-phonon coupling strength through the lanthanide series. The combination of the two ef- 
fects, lanthanide contraction and shielding, with an opposite influence on the electron-phonon coupling strength can explain the symmetric behaviour around the half-filled shell configuration. The first effect, lanthanide contraction, is well established. For the second eflect, the shielding factor for $4 f$ electrons, different values have been reported. For example, Newman and Price have calculated a much higher shielding factor for $\mathrm{Gd}^{3+}$. Intuitively, a high shielding factor can be expected for the half-filled shell configuration $4 f^{7}$. A high shielding factor for the $4 f$ electrons is in agreement with the very weak electron-phonon coupling observed for $\mathrm{Gd}^{3+}$.

The $\bar{\alpha}$ values for $\mathrm{Tb}^{3+}$ are higher than expected. To explain this observation, the position of the $4 f^{n-1} 5 d$ states has to be considered (Fig. 5). Between $\mathrm{Gd}^{3+}$ and $\mathrm{Tb}^{3+}$ there is a large discontinuity. The much lower position of the $4 f^{n-1} 5 d$ state for $\mathrm{Tb}^{3+}$ explains the relatively high value for the electron-phonon coupling strength. Due to the low energy of the $4 f^{n-1} 5 d$ state, the admixing of this opposite parity state in to the $4 f^{n}$ states is larger which gives rise to stronger electron-phonon coupling.

TABLE II

Electron-phonon coupling parameters $\vec{\alpha}$ for $\mathrm{Pr}^{3+}, \mathrm{Nd}^{3+}$ and $\mathrm{Gd}^{3+}$ in $\mathrm{LiYF}_{4}$ and $\mathrm{La}_{2} \mathrm{O}_{3}$.

\begin{tabular}{c|c|c}
\hline \hline $\begin{array}{c}\text { Tramsition } \\
\mathrm{P}_{1}{ }^{3+}\end{array}$ & $\begin{array}{c}\mathrm{LiYF}_{4} \\
\bar{\alpha}\left[\mathrm{cm}^{-1}\right]\end{array}$ & $\begin{array}{c}\mathrm{La}_{2} \mathrm{O}_{3} \\
\bar{\alpha}\left[\mathrm{cm}^{-1}\right]\end{array}$ \\
\hline${ }^{3} P_{0} \rightarrow{ }^{3} H_{4}$ & 80 & 100 \\
${ }^{3} P_{0} \rightarrow{ }^{3} H_{6}$ & 125 & 210 \\
${ }^{1} D_{2} \rightarrow{ }^{3} H_{4}$ & 160 & 500 \\
\hline $\mathrm{Nd}^{3+}$ & & \\
\hline${ }^{3} P_{3 / 2} \rightarrow{ }^{4} I_{11 / 2}$ & 50 & 140 \\
\hline $\mathrm{Grd}^{3+}$ & & \\
\hline${ }^{6} P_{7 / 2} \rightarrow{ }^{5} S_{7 / 2}$ & 3 & 12 \\
${ }^{6} I_{T / 2} \rightarrow{ }^{8} S_{7 / 2}$ & - & 8
\end{tabular}

To study the influence of the host lattice on the electron-phonon coupling strength, line broadening was measured for $\mathrm{Pr}^{3+}, \mathrm{Nd}^{3+}$ and $\mathrm{Gd}^{3+}$ in $\mathrm{La}_{2} \mathrm{O}_{3}$. In Table II the results of fits to Eq. (2) for the temperature dependence of the line width are given for transitions between the same multiplets for $\mathrm{Pr}^{3+}, \mathrm{Nd}^{3+}$ and $\mathrm{Gd}^{3+}$ in $\mathrm{LiYF}_{4}$ and $\mathrm{La}_{2} \mathrm{O}_{3}$. In all cases stronger coupling (higher $\bar{\alpha}$ values) are found for rare earth ions in $\mathrm{L}_{42} \mathrm{O}_{3}$. This observation is in agreement with the data on vibronic transition probabilities reported above. An increase in covalency in the host lattice can be considered as an increase in the spatial extension of the electron wave functions of the rare earth ions and the ligands. The larger spatial extension of the $4 f$ orbitals and the ligands enhances the electron-phonon coupling strength. 


\section{Consequences}

Now that we understand how the electron-phonon coupling strength varies through the series of rare earth ions and how it depends on the covalency of the host lattice, it is interesting to see how this large variation in electron-phonon coupling strength manifests itself in phenomena like multiphonon relaxation, (phonon-assisted) energy transfer and processes which involve interaction between neighbouring rare earth ions. All these processes are expected to be most efficient for the light and heavy lanthanides in covalent host lattices. For (phonon-assisted) energy transfer there is a large number of data in literature on transfer rates between different rare ions in different host lattices. Unfortunately, a comparison of the transfer rates to see the effect of the variation of the electron-phonon coupling strength on the transfer rates is difficult. Other factors, like differences in the energy mismatch, also influence the transfer rate. A second complication is that different methods of analysis are used to derive transfer rates and this seems to influence the values which are lound for the transfer rates. Still, the effect of covalency is well known: energy transfer is more efficient in covalent host lattices than in ionic host lattices [14].

A nice example of a process which involves the interaction between two neighbouring rare earth ions is cooperative luminescence. This effect has been observed for the first time for $\mathrm{Yb}^{3+}$ in $\mathrm{YbPO}_{4}[15]$. Excitation in the ${ }^{2} F_{5 / 2}$ level of $\mathrm{Yb}^{3+}$ at about $10000 \mathrm{~cm}^{-1}$ yields a weak emission in the green at exactly the double energy of the ${ }^{2} F_{5 / 2} \rightarrow{ }^{2} F_{7 / 2}$ transition of $\mathrm{Yb}^{3+}$. The weak green emission is explained by the cooperative transition of two neighbouring $\mathrm{Yb}^{3+}$ ions in the excited state to the ground state. After the first observation, cooperative luminescence has also been observed for $\mathrm{Yb}^{3+}$ in other host lattices with $\mathrm{Yb}^{3+}$ concentrations down to $10 \%$ [16]. Recently cooperative luminescence was observed for a very low concentration of $\mathrm{Yb}^{3}+$ in $\mathrm{La}_{2} \mathrm{O}_{3}(0.5 \%)$ [17]. With the present knowledge on how the interaction of the $4 f$ electrons of rare earth ions with their environment depends on the type of rare earth ion and the covalency of the host lattice, this observation can be understood. For the heavy rare earth ion $\mathrm{Yb}^{3+}$ in a covalent host lattice $\left(\mathrm{La}_{2} \mathrm{O}_{3}\right)$ a strong coupling is expected. This explains the observation of cooperative luminescence for such a low $\mathrm{Yb}^{3+}$ concentration.

A third phenomenon where the influence of the electron-phonon coupling is expected is multiphonon relaxation. For stronger electron-phonon coupling, faster multiphonon relaxation is expected. The most widely used model for multiphonon relaxation, the energy gap model, does not take a variation of the electron-phonon coupling strength into account: the relaxation rate only depends on the energy gap and the maximum phonon energy. For various host lattices (crystals and glasses) doped with different rare earth ions the exponential energy gap law has been shown to be a good approximation [18]. The fact that the variation of the electron-phonon coupling strength is not clearly observed is probably due to the fact that the multiphonon relaxation rates are plotted on a logarithmic scale and that usually values for different ions in the same host lattice are compared. To study if the influence of the variation in electron-phonon coupling strength can be observed in multiphonon relaxation rates, we studied the multiphonon relaxation for $\mathrm{Gd}^{3+}$ 
(middle) and $\mathrm{Nd}^{3+}$ (light) in $\mathrm{LiYF}_{4}$ (ionic) and $\mathrm{La}_{2} \mathrm{O}_{3}$ (covalent). The maximum phonon energy in the two host lattices is similar (about $500 \mathrm{~cm}^{-1}$ ) and for both rare earth ions multiphonon relaxation rates were measured over energy gap of about $2000 \mathrm{~cm}^{-1}\left({ }^{6} I_{7 / 2} \rightarrow{ }^{6} P_{3 / 2}\right.$ for $\mathrm{Gd}^{3+}$ and ${ }^{2} P_{3 / 2} \rightarrow{ }^{4} D_{5 / 2}$ for $\left.\mathrm{Nd}^{3+}\right)$. Based on the energy gap law, the multiphonon relaxation rates should be similar for all four cases since energy gaps and the maximum phonon frequencies are similar. Based on the variation in electron-phonon coupling strength, the fastest multiphonon relaxation is expected for $\mathrm{Nd}^{3+}$ in $\mathrm{La}_{2} \mathrm{O}_{3}$ and the slowest for $\mathrm{Gd}^{3+}$ in $\mathrm{LiYF}_{4}$. To determine the multiphonon relaxation rates the temperature dependence of the decay time of the higher level $\left({ }^{6} I_{7 / 2}\right.$ for $\mathrm{Gd}^{3+}$ and ${ }^{2} P_{3 / 2}$ for $\mathrm{Nd}^{3+}$ ) was measured. The results were fitted to

$$
\frac{1}{\tau}=A_{\mathrm{R}}+A_{\mathrm{NR}}(T)
$$

where

$$
A_{\mathrm{NR}}(T)=(n+1)^{p} A_{\mathrm{NR}}^{\mathrm{MPR}}(0) .
$$

\section{TABLE III}

Multiphonon relaxation rates for the ${ }^{6} I_{7 / 2} \rightarrow{ }^{6} P_{3 / 2}$ transition on $\mathrm{Gd}^{3+}$ and the ${ }^{2} P_{3 / 2} \rightarrow{ }^{4} D_{5 / 2}$ transition on $\mathrm{Nd}^{3+}$ in $\mathrm{LiYF}_{4}$ and $\mathrm{La}_{2} \mathrm{O}_{3}$ are shown. The parameters for the best fits to Eq. (3) are given, together with typical $\bar{\alpha}$ values derived from temperature dependent line broadening measurements on the same systems.

\begin{tabular}{l|c|c|c|c|c}
\hline \hline Lattice & Ion & $p$ & $\hbar \omega\left[\mathrm{cm}^{-1}\right]$ & $A_{\mathrm{NR}}^{\mathrm{MPR}}(0)$ & $\bar{\alpha}\left[\mathrm{cm}^{-1}\right]$ \\
\hline $\mathrm{LiYF}_{4}$ & $\mathrm{Gd}^{3+}$ & 5 & 440 & 680 & 3 \\
& $\mathrm{Nd}^{3+}$ & 6 & 415 & 9300 & 50 \\
$\mathrm{La}_{2} \mathrm{O}_{3}$ & $\mathrm{Gd}^{3+}$ & 5 & 433 & 2510 & 12 \\
& $\mathrm{Nd}^{3+}$ & 5 & 443 & $2.8 \times 10^{5}$ & 140
\end{tabular}

Here $A_{\mathrm{R}}$ is the radiative decay rate (assumed to be temperature independent), $A_{\mathrm{NR}}(T)$ is the multiphonon relaxation rate, $n$ is the phonon occupation number for the highest energy phonons and $p=\Delta E / \hbar \omega$, the total number of phonons involved in the multiphonon relaxation process. The results of the fits are shown in Table III. For Gd ${ }^{3+}$ the multiphonon relaxation rates $A_{\mathrm{NR}}^{\mathrm{MPR}}(0)$ were also estimated from the ratio of the emission intensities from the ${ }^{6} P_{7 / 2}$ and ${ }^{6} I_{7 / 2}$ level at $4 \mathrm{~K}$. The rates determined from the relative intensities of the emission from the two levels are in good agreement with those in Table III. For $\mathrm{Nd}^{3+}$ it was not possible to measure the relative emission intensities from the two levels because part of the emission is in the infrared and there is an overlap between emission lines from the two levels. This makes the values reported for the multiphonon relaxation rates for $\mathrm{Nd}^{3+}$ less ireliable. The large variation in multiphonon relaxation rates for the four systems shows that the multiphonon relaxation does indeed depend on the electron-phonon coupling strength. For comparison $\bar{\alpha}$ values which were found 
for the four systems are included in Table III. The multiphonon relaxation rates vary in the same way as the $\bar{\alpha}$ values: the larger $\bar{\alpha}$ is, the higher the multiphonon relaxation rates are. To establish a more convincing relationship between $\bar{\alpha}$ and multiphonon relaxation rates, a more detailed study of a much larger number of transitions is required.

\section{Conclusions}

The variation of the electron-phonon coupling strength for rare earth ions is large. Measurements on vibronic transition probabilities and temperature dependent line width measurements show that the electron-phonon coupling strength is larger for the light and heavy rare earth ions, and smaller for ions in the middle. This variation can be understood qualitatively by two effects with an opposite influence on the electron-phonon coupling strength: lanthanide contraction and the shielding of the $4 f$ electrons by the $5 s$ and $5 p$ electrons. The coupling is also influenced by the host lattice: in a covalent host lattice the coupling is stronger. The difference in electron-phonon coupling strengths has important consequences in processes that involve the interaction of the $4 f$ electrons and their surroundings.

\section{Acknowledgment}

The authors are grateful to Mr. M.L.H. ter Ileerdt, Mr. II. Anders and Mr. R.T. Wegh for performing part of the measurements. The measurements on $\mathrm{Y}^{3} \mathrm{~b}^{3+}$ were performed in the group of Prof. II.U. Güdel (Bern, Switzerland) and we are especially indebted to Prof. II.U. Güdel and Dr. T. Riedener for their help and suggestion to measure the cooperative luminescence. The investigations were supported by the Netherlands Foundation for Chemical Research (SON) with financial aid from the Netherlands Organization for Scientific Research (NWO).

\section{References}

[1] K.H. Hellwege, Ann. Phys. 40, 529 (1941).

[2] W.F. Krupke, Phys. Rev. 145, 325 (1966).

[3] R.D. Peacock, in: Structure and Bonding, Eds. J.D. Dunitz, P. Hemmerich, R.H. Holm, J.A. Ibers, C.K. Jorgensen, J.B. Neilands, D. Reinen, R.J.P. Williams, Vol. 22, Springer Verlag, Berlin 1975, p. 84.

[1] L.A. Riseberg, H.W. Moos, Phys. Rev. 174, 429 (1968).

[5] J.T. Gourley, Phys. Rev. B 5, 22 (1972).

[6] J. Sytsma, W. van Schaik, G. Blasse, J. Phys. Chem. Solids 52, 419 (1991).

[7] C. de Mello Donega, A. Meijerink, G. Blasse, J. Phys. Condens. Matter 4, 8889 (1992).

[8] A. Ellens, S. Schenker, A. Meijerink, G. Blasse, J. Lumin., accepted for publication.

[9] A. Ellens, H. Andres, H.L.H. ter Heerdt, R.T. Wegh, A. Meijerink, G. Blasse, to be published.

[10] B. Henderson, G.F. Imbusch, Optical Spectroscopy of Inorganic Solids, Clarendon Press, Oxford 1989, p. 233.

[11] W.M. Yen, W.C. Scott, A.L. Shawlow, Phys. Rev. 136, 271 (1964). 
[12] N. Kushida, M. Kikucli, J. Phys. Soc. Japan 23, 1333 (1967).

[13] T. Szczurek, M. Schllesinger, in: Rare Earth Spectroscopy, Eds. B. Jeżowska-Trzebiatowska, J. Legendziewicz, W. Stręk, World Scientific, Singapore 1985, p. 325.

[14] A.J. de Vries, H.S. KRiliaan, G. Blasse, J. Solid State Chem. 65, 190 (1986).

[15] E. Nakazawa, S. Shionoya, Phys. Rev. Lett. 25, 1710 (1970).

[16] M.P. Hehlen, H.U. Güdel, J. Phys. Chem. 98, 1768 (1993).

[17] R.T. Wegh, A. Meijerink, Chem. Phys. Lett. 246, 495 (1995).

[18] I.A. Riseberg, M.J. Weber, in: Progress in Optics, Ed. E. Wolf, North-Holland, Amsterdam 1976, p. 89. 